\title{
Erythropoietin protects lipopolysaccharide-induced renal mesangial cells from autophagy
}

\author{
LINGYUN BI ${ }^{1}$, RUANLING HOU ${ }^{2}$, DASHENG YANG ${ }^{1}$, SHUJUN LI $^{1}$ and DEAN ZHAO ${ }^{1}$ \\ ${ }^{1}$ Department of Pediatrics, The First Affiliated Hospital of Xinxiang Medical University, Weihui, Henan 453100; \\ ${ }^{2}$ Physiology Laboratory, Xinxiang Medical University, Xinxiang, Henan 453000, P.R. China
}

Received March 18, 2014; Accepted October 28, 2014

DOI: 10.3892/etm.2014.2124

\begin{abstract}
The aim of this study was to investigate the effects of erythropoietin (EPO) on the impairment of autophagy induced by lipopolysaccharide (LPS) in primary cultured rat glomerular mesangial cells (GMCs). Rat GMCs were isolated and cultured in normal glucose, high-glucose, LPS or LPS + EPO medium. At 24 and $72 \mathrm{~h}$ of culture, the cells were examined for expression levels of the autophagy markers LC3 and p62/sequestosome-1 (SQSTM1) using western blot analysis. At $24 \mathrm{~h}$, no significant difference in the expression of LC3 and p62/SQSTM1 was observed among the groups; however, the cells exposed to high-glucose medium for $72 \mathrm{~h}$ showed downregulated LC3 expression and upregulated p62/SQSTM1 expression. The cells exposed to LPS $(10 \mathrm{ng} / \mathrm{ml})$ for $72 \mathrm{~h}$ showed upregulated LC3 expression and upregulated p62/SQSTM1 expression. These changes were reversed in the LPS + EPO group at $72 \mathrm{~h}$. In conclusion, EPO can inhibit LPS-induced autophagy in rat GMCs.
\end{abstract}

\section{Introduction}

Autophagy exists in higher eukaryotes ranging from yeast to humans. The process is highly conserved and maintains intracellular stability (1). Distinct from the ubiquitin-proteasome pathway, which degrades short-life proteins, autophagy degrades long-life bioplasmin and malfunctional organelles. There a five steps in the autophagy process (2-4): i) Activation of a dual-membrane structure, known as a phagocytic vacuole; ii) amplification of the phagocytic vacuole; iii) maturation of the phagosome; iv) combination between phagosome and lysosome; v) autophagy. In normal physiological conditions, autophagy is maintained at a low level and preserves normal physiological function by cleaning up injured organelles and biomolecules, such as mitochondria and proteins. Dysfunctional autophagy

Correspondence to: Professor Dasheng Yang, Department of Pediatrics, The First Affiliated Hospital of Xinxiang Medical University, 88 Jiankang Road, Weihui, Henan 453100, P.R. China E-mail: dashengyang@yeah.net

Key words: erythropoietin, renal mesangial cells, autophagy, p62/sequestosome-1 can lead to cancer and degenerative disease; however, whether autophagy participates in the pathogenesis of kidney disease remains unclear. Considerable evidence shows a close association between autophagy and a number of kidney diseases, including acute kidney injury (AKI), diabetic nephropathy and polycystic kidney disease (5). This association could be utilized in the development of novel treatments for kidney diseases.

Erythropoietin (EPO) is a hormone that regulates the generation of erythrocytes. It is primarily produced by the renal cortex and peritubular fibroblasts. The levels of renal, hepatic and cerebral endogenous EPO increase when blood supply or oxygen insufficiency occurs (6). EPO assists oxygen delivery and erythropoiesis and reduces apoptosis, oxidative stress and inflammation $(7,8)$. Several studies have attempted to show the effects of EPO on the kidney, but the mechanism has remained unclear (Jackevicius, Moeini).

The precursor LC3 is divided and formed into LC3-I by autophagy-related gene 4 (Atg4). LC3-I is transformed into the membrane-bound form LC3-II through the activation of $\operatorname{Atg} 7$, which is located on the membrane of autophagosomes and autolysosomes (9). LC3-I and LC3-II are indicators of autophagy, and show the activation of autophagy (10). p62/sequestosome-1 (SQSTM1) is an ubiquitin-binding protein, and can induce the autophagy of ubiquitinated proteins by lysosomes in mammalian cells, through cooperation with LC3 (11). The high expression of p62/SQSTM1 can cause an upregulation of the autophagy process and can act as an indicator of autophagy (12).

The aim of the present study was to induce autophagy in rat renal glomerular mesangial cells (GMCs) through the use of lipopolysaccharide (LPS) and to explore the effect of EPO on GMC autophagy to provide novel insight into EPO-mediated renal protection.

\section{Materials and methods}

Isolation and culture of rat renal GMCs. A total of 32 two-month-old male Sprague Dawley rats were supplied by the Henan Laboratory Animal Center (Zhengzhou, China). This study was carried out in strict accordance with the recommendations in the Guide for the Care and Use of Laboratory Animals of the National Institutes of Health. The animal use protocol was reviewed and approved by the Institutional Animal Care and Use Committee of the First 
Affiliated Hospital of Xinxiang Medical University (Weihui, China). Both kidneys were isolated from the two-month-old Sprague Dawley rats aseptically. The renal capsule was exfoliated, minced into small pieces and placed on a three-layered stainless steel screen, prior to being washed and ground with a pestle simultaneously. The washing process was terminated when kidney tubules could no longer be observed under the microscope and when $>98 \%$ of the glomeruli were not associated with the Bowman's capsule. The glomerular tissue was then collected from the second screen $(<75 \mu \mathrm{m})$ into a centrifuge tube for centrifugation at $15,000 \mathrm{x}$ g for $5 \mathrm{~min}$. The supernatant was subsequently discarded and the sediment was treated with collagenase V (Sigma, St. Louis, MO, USA) for $10-15 \mathrm{~min}$ at $37^{\circ} \mathrm{C}$. The reaction was terminated with the addition of RPMI-1640 medium $/ 15 \%$ fetal bovine serum (FBS) (Gibco-BRL, Grand Island, NY, USA). The sample was then centrifuged at $15,000 \mathrm{x} \mathrm{g}$ for a further 5 min prior to the supernatant being discarded. The glomeruli were collected and seeded in a gelatinized culture flask with RPMI-1640 medium $/ 15 \% \mathrm{FBS}$ at $37^{\circ} \mathrm{C}$ and $5 \% \mathrm{CO}_{2}$. The first passage was conducted after 7-10 days. Twenty-four hours after passage, the cells adhered and formed fusiform or irregular stellate cells. After 3-4 days of culture, the cells formed a tablet.

Immunofluorescent staining. Immunofluorescent staining was performed by initially activating the cells in vitro, staining the surface for antigens and fixing with paraformaldehyde in order to stabilize the cell membrane. The cell membrane was then permeabilized with the detergent saponin in order to allow the antibodies to stain intracellularly. Immunofluorescence showed positive results for monoclonal mouse anti-human $\alpha$-smooth muscle actin (1:200 dilution; Beyotime Institute of Biotechnology, Shanghai, China) and rabbit anti-human monoclonal vimentin (1:200 dilution; Beyotime Institute of Biotechnology), and negative results for mouse monoclonal cytokeratin (1:200 dilution; Beyotime Institute of Biotechnology) and mouse monoclonal factor VIII (1:200 dilution; Beyotime Institute of Biotechnology). Cells cultured over three passages were used for examination.

Grouping. The cells were divided into four groups as follows: i) Control (cells were cultured in RPMI-1640 medium/15\% FBS and $5 \mathrm{mmol} / \mathrm{l}$ glucose; ii) high-glucose (cells were cultured in RPMI-1640 medium/15\% FBS and $30 \mathrm{mmol} / 1$ glucose; iii) LPS (cells were cultured in RPMI-1640 medium/15\% FBS and $10 \mathrm{nmol} / \mathrm{ml}$ LPS (Sigma); iv) LPS + EPO (cells were cultured in RPMI-1640 medium/15\% FBS, $10 \mathrm{ng} / \mathrm{ml}$ LPS and $10 \mathrm{nmol} / \mathrm{ml}$ EPO (Sigma). Cells were harvested for examination after 24- and 72-h culture periods.

Western blot analysis. A total of $0.1 \mathrm{ml}$ pre-chilled radioimmunoprecipitation assay buffer $[50 \mathrm{mmol} / \mathrm{l}$ Tris- $\mathrm{Cl}(\mathrm{pH} 7.6)$, $150 \mathrm{mmol} / 1 \mathrm{NaCl}, 1 \% \mathrm{NP}-40,0.1 \%$ sodium dodecyl sulfate (SDS), $0.5 \%$ deoxycholate, $1 \mu \mathrm{l} / \mathrm{ml}$ leupeptin, $1 \mu \mathrm{l} / \mathrm{ml}$ aprotinin and $0.5 \mathrm{mmol} / \mathrm{l}$ phenylmethylsulfonyl fluoride] (Beyotime Institute of Biotechnology) was added to the homogenate and chilled on ice for $30 \mathrm{~min}$. The sample was then centrifuged at $15,000 \mathrm{x} \mathrm{g}$ for $30 \mathrm{~min}$, the supernatant was pipetted and the

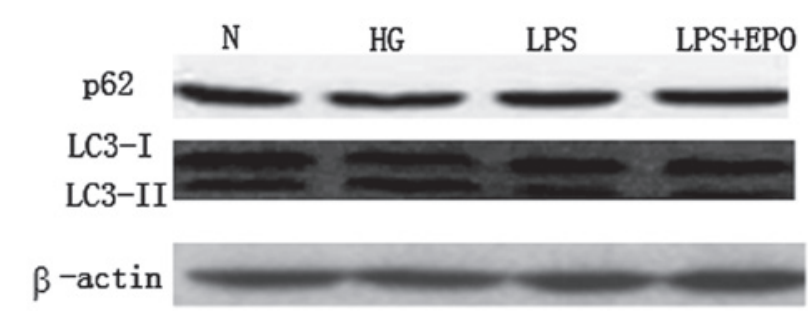

Figure 1. Western blot analysis of LC3 and p62/sequestosome-1 showed no significant differences among the groups after $24 \mathrm{~h}$ of culture. N, normal control; HG, high-glucose; LPS, lipopolysaccharide; EPO, erythropoietin.

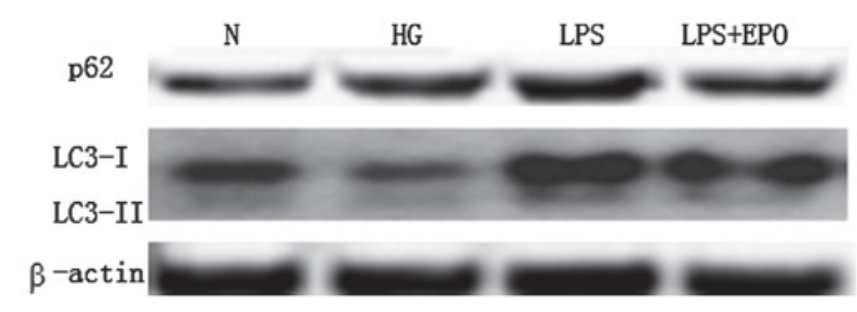

Figure 2. After $72 \mathrm{~h}$ of culture, p62/SQSTM1 expression was increased and LC3 expression was decreased in the HG group compared with that in the $\mathrm{N}$ group. By contrast, p62/SQSTM1 expression was decreased and LC3 expression was increased in the LPS group. The LPS-induced changes were reversed in the LPS + EPO group. SQSTM1, sequestosome-1; N, normal control; HG, high-glucose; LPS, lipopolysaccharide; EPO, erythropoietin.

concentration of protein was measured with a bicinchoninic acid protein assay kit (Baiwang Biotechnology Corporation, Shenzhen, China). SDS loading buffer $(10 \mathrm{ml})$ [2X; $2 \mathrm{ml}$ $0.5 \mathrm{~mol} / 1$ Tris (pH 6.8), $2 \mathrm{ml}$ glycerin, $2 \mathrm{ml} \mathrm{20 \%} \mathrm{SDS,}$ $0.5 \mathrm{ml} 0.1 \%$ bromophenol blue, $1 \mathrm{ml} 1 \mathrm{~mol} / 1$ dithiothreitol

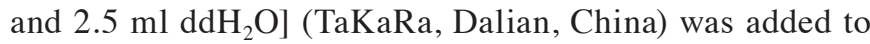
$30-50 \mu \mathrm{g}$ protein, and the sample was heated at $100^{\circ} \mathrm{C}$ for $5 \mathrm{~min}$ to denature the protein. Following SDS-PAGE and protein transfer to a nitrocellulose membrane, the membrane was blocked with $1 \mathrm{X}$ blocking buffer [5\% skimmed milk powder $0.5 \mathrm{~g} ; 20 \mathrm{mM}$ Tris-Cl, (pH 7.5-8.0); $150 \mathrm{mM} \mathrm{NaCl}$; 0.05\% Tween-20; Baiwang Biotechnology Corporation] at room temperature for $1 \mathrm{~h}$. The membrane was then immersed in the primary antibody (LC3, dilution ratio 1:2,000; p62, dilution ratio 1:200) (Santa Cruz Biotechnology, Inc., Santa Cruz, CA, USA) and incubated overnight at $4^{\circ} \mathrm{C}$. Following incubation with the polyclonal primary antibody (catalogue no. F2426), the membrane was rinsed three times with Tris-buffered saline and Tween ${ }^{\circledR} 20$ (Baiwang Biotechnology Corporation), with each rinse lasting $5 \mathrm{~min}$. The membrane was then immersed in mouse anti-rabbit secondary antibody (dilution ratio 1:2,000) and incubated at room temperature for $1 \mathrm{~h}$, prior to being rinsed a further three times. A chemiluminescent graph was used to be colored and detected. All of the experiments were performed according to the manufacturer's instructions (Baiwang Biotechnology Corporation). $\beta$-actin was used as an internal control.

Statistical analysis. All data are expressed as the mean \pm standard deviation. Comparisons between two groups were conducted with an independent samples t-test. SPSS 17.0 (SPSS, Inc., Chicago, IL, USA) was utilized for the analysis. $\mathrm{P}<0.05$ was considered to indicate a statistically significant difference. 


\section{Results}

In the present study, no significant differences in the protein expression of LC3-I and II, and p62/SQSTM1 were found among the four groups in the 24-h culture experiment (Fig. 1). After $72 \mathrm{~h}$ of culture, the expression of p62/SQSTM1 was increased, and that of LC3-I and II was decreased in the high-glucose group compared with the expression in the control group (Fig. 2). By contrast, the expression of p62/SQSTM1 was increased and that of LC3-I and II was increased in the LPS group. The changes were reversed in the LPS + EPO group (Fig. 2). These results suggest that high-glucose conditions can inhibit autophagy in mesangial cells; LPS can induce autophagy in mesangial cells and EPO can suppress this induction.

\section{Discussion}

Several factors, such as diabetes and bacterial infection, can lead to a renal disease, which can seriously affect human health. Autophagy is a common defense mechanism that exists widely in eukaryotes. Autophagy can maintain the intracellular stability by cleaning up damaged or aged organelles and biomolecules, such as mitochondria, peroxisomes, endoplasmic reticulum and paraprotein $(13,14)$. Autophagy therefore plays an important role in pathology. It has been shown that autophagy participates in renal ischemia/reperfusion injury. In a study by Li et al (15) it was suggested that high glucose concentrations could inhibit autophagy in mesangial cells, while rapamycin could suppress the high glucose-induced autophagy and N3-methyladenine could upregulate the high glucose-induced autophagy in renal mesangial cells. Renal diseases usually cause anemia, due to an EPO deficiency originating from the renal cortex and renal tubule fibroblasts. As a result, EPO is often administered in renal therapy. In a recent study, Yamaleyeva et al (16) treated chronic renal injury with EPO-positive cells and revealed promising results. Furthermore, in a study by Oh et al (17) it was found that kidney function could be conditioned using EPO following the occurrence of AKI (17), mainly by improving oxygen transport and erythropoiesis, as well as reducing autophagy, oxidative stress and inflammation $(7,8)$.

In the present study, it was found that high-glucose conditions could suppress autophagy in GMCs; this finding was consistent with the results in the study by Li et al (15). The present results suggested that LPS could induce autophagy in GMCs, and that EPO could inhibit the LPS-induced autophagy and protect the renal cells from damage. In an oxidative toxicity rat model, EPO was previously shown to block the autophagy signals in the brain to keep the brain from damage (18). In a recent study by Yu et al (19), it was reported that EPO could protect endothelial cells through the same mechanism in a neonatal necrotizing enterocolitis rat model. This indicated that inflammation could induce autophagy and apoptosis and that EPO could block this pathway to protect the cells. The inhibition of autophagy has certain value in clinical practice. A previous study by Zhang et al (20) showed that the treatment of folliculin-deficient renal cancer was enhanced through the use of a combination paclitaxel and autophagy inhibitor treatment. Both autophagy inhibition and efforts to target the B-cell lymphoma-2 family of proteins are strategies that could overcome drug tolerance (21).
By contrast, Kimura et al (22) reported that mitochondrial metabolic stress in renal proximal tubular epithelial cells can lead to a defensive autophagy. Furthermore, the study by Yamahara et al (23) showed that obesity-mediated autophagy cannot exacerbate proteinuria-induced tubulointerstitial injury. The role of autophagy in renal disease therefore remains to be fully elucidated.

In conclusion, the present study showed that high-glucose conditions can inhibit autophagy in renal cells, and that LPS can induce autophagy. Furthermore, EPO can reverse this LPS-induced autophagy and protect renal mesangial cells from damage.

\section{References}

1. Levine B and Klionsky DJ: Development by self-digestion: molecular mechanisms and biological functions of autophagy. Dev Cell 6: 463-477, 2004.

2. Yang $\mathrm{Z}$ and Klionsky DJ: Eaten alive: a history of macroautophagy. Nat Cell Biol 12: 814-822, 2010.

3. Weide $\mathrm{T}$ and Huber TB: Implications of autophagy for glomerular aging and disease. Cell Tissue Res 343: 467-473, 2011.

4. Mizushima N and Levine B: Autophagy in mammalian development and differentiation. Nat Cell Biol 12: 823-830, 2010.

5. Huber TB, Edelstein CL, Hartleben B, et al: Emerging role of autophagy in kidney function, diseases and aging. Autophagy 8 : 1009-1031, 2012.

6. Weidemann A and Johnson RS: Nonrenal regulation of EPO synthesis. Kidney Int 75: 682-688, 2009.

7. Toba H, Nakashima K, Oshima Y, et al: Erythropoietin prevents vascular inflammation and oxidative stress in subtotal nephrectomized rat aorta beyond haematopoiesis. Clin Exp Pharmacol Physiol 37: 1139-1146, 2010.

8. Malgorzewicz S, Lichodziejewska-Niemierko M, Lizakowski S, et al: Oxidative stress, inflammation and nutritional status during darbepoetin alpha treatment in peritoneal dialysis patients. Clin Nephrol 73: 210-215, 2010.

9. Maiuri MC, Zalckvar E, Kimchi A and Kroemer G: Self-eating and self-killing: crosstalk between autophagy and apoptosis. Nat Rev Mol Cell Biol 8: 741-752, 2007.

10. Kadowaki M and Karim MR: Cytosolic LC3 ratio as a quantitative index of macroautophagy. Methods Enzymol 452: 199-213, 2009.

11. Pankiv S, Clausen TH, Lamark T, et al: p62/SQSTM1 binds directly to Atg8/LC3 to facilitate degradation of ubiquitinated protein aggregates by autophagy. J Biol Chem 282: 24131-24145, 2007.

12. Mizushima $\mathrm{N}$ and Yoshimori T: How to interpret LC3 immunoblotting. Autophagy 3: 542-545, 2007.

13. Kondo-Okamoto N, Noda NN, Suzuki SW, et al: Autophagy-related protein 32 acts as an autophagic degron and directly initiates mitophagy. J Biol Chem 287: 10631-10638, 2012.

14. Xie Z and Klionsky DJ: Autophagosome formation: core machinery and adaptations. Nat Cell Biol 9: 1102-1109, 2007.

15. Li J, Bai XY, Cui SY, Fu B and Chen X: Effect of rapamycin on high glucose-induced autophagy impairment, oxidative stress and premature senescence in rat mesangial cells in vitro. Nan Fang Yi Ke Da Xue Xue Bao 32: 467-471, 2012 (In Chinese).

16. Yamaleyeva LM, Guimaraes-Souza NK, Krane LS, et al: Cell therapy with human renal cell cultures containing erythropoietin-positive cells improves chronic kidney injury. Stem Cells Transl Med 1: 373-383, 2012.

17. Oh SW, Chin HJ, Chae DW and Na KY: Erythropoietin improves long-term outcomes in patients with acute kidney injury after coronary artery bypass grafting. J Korean Med Sci 27: 506-511, 2012.

18. Bendix I, Schulze C, Haefen Cv, et al: Erythropoietin modulates autophagy signaling in the developing rat brain in an in vivo model of oxygen-toxicity. Int J Mol Sci 13: 12939-12951, 2012.

19. Yu Y, Shiou SR, Guo Y, et al: Erythropoietin protects epithelial cells from excessive autophagy and apoptosis in experimental neonatal necrotizing enterocolitis. PLoS One 8: e69620, 2013. 
20. Zhang Q, Si S, Schoen S, et al: Suppression of autophagy enhances preferential toxicity of paclitaxel to folliculin-deficient renal cancer cells. J Exp Clin Cancer Res 32: 99, 2013.

21. Mei H, Lin Z, Wang Y, Wu G and Song Y: Autophagy inhibition enhances pan-Bcl-2 inhibitor AT-101-induced apoptosis in non-small cell lung cancer. Neoplasma 61: 186-192, 2014.
22. Kimura T, Takahashi A, Takabatake Y,et al: Autophagy protects kidney proximal tubule epithelial cells from mitochondrial metabolic stress. Autophagy 9: 1876-1886, 2013.

23. Yamahara K1, Kume S, Koya D, et al: Obesity-mediated autophagy insufficiency exacerbates proteinuria-induced tubulointerstitial lesions. J Am Soc Nephrol 24: 1769-1781, 2013. 